\title{
AUTO-ORGANIZAÇÃO E OS IDOSOS: A INTERLOCUÇÃO NECESSÁRIA ATRAVÉS DE UM FÓRUM
}

\author{
Leticia Rocha Machado \\ Universidade Federal do Rio Grande do Sul. Programa de Pós Graduação em Informática na Educação \\ leticiarmachado@yahoo.com.br \\ Anelise Jantsch \\ Universidade Federal do Rio Grande do Sul. Programa de Pós Graduação em Informática na Educação \\ anelise.jantsch@gmail.com \\ Johannes Doll \\ Universidade Federal do Rio Grande do Sul. Programa de Pós Graduação em Educação \\ johannes.doll@ufrgs.br \\ Patricia Alejandra Behar \\ Universidade Federal do Rio Grande do Sul. Programa de Pós Graduação em Informática na Educação \\ pbehar@terra.com.br \\ José Valdeni Lima \\ Universidade Federal do Rio Grande do Sul. Programa de Pós Graduação em Informática na Educação \\ valdeni@inf.ufrgs.br
}

RESUMO: O presente artigo apresenta como o recurso fórum pôde propiciar um processo de aprendizagem a luz da teoria da auto-organização. Para tanto, foi utilizado na coleta de dados o AVA ROODA em um curso para idosos desenvolvido na UFRGS. Por meio da análise de 21 postagens realizadas por 16 idosos ao longo do curso constatou-se que o processo de escrita de narrativas levou à reflexão, mudança de comportamento e adoção de atitudes que contemplam a qualidade de vida, ou em outras palavras, ao processo de envelhecimento ativo.

Palavras-chaves: auto-organização, idosos, tecnologias de informação e comunicação.

\section{SELF-ORGANIZATION AND THEELDERLIES: AREQUIREDINTERLOCUTIONTHROUGH AFORUM}

\begin{abstract}
This paper shows how the resource forum could provide a process of learning second the theory of self-organization. For this purpose, was used to collect data the AVA ROODA on a course designed for seniors at UFRGS. Through analysis of 21 posts made of 16 elderlies over the course we found that the process of writing narratives led to reflection, behavior changes and adoption of attitudes that include quality of life, or in other words, the process active ageing.
\end{abstract}

Keywords: self-organization, elderlies, information and communication technologies.

\section{INTRODUÇÃO}

Além de um processo demográfico que já se conhece, o envelhecimento pressupõe vários aspectos biológicos, psicológicos e sociais que muitas vezes são 
desconsiderados em uma análise mais detalhada na educação permanente. Entender este processo é compreender os aspectos individuais e coletivos da vida (Vitola, Argimon, 2003; Osório, 2007).

Neste contexto evidencia-se a necessidade de realizar discussões sobre a qualidade de vida que se deseja para a população que envelhece. As intervenções educativas possuem múltiplas possibilidades para se trabalhar os aspectos biopsicossociais do envelhecer. Neste sentido, é cada vez mais necessário o estudo da teoria da auto-organização em um mundo que despreza o "eu" e a relação com o "outro" e com o "seu eu" no intuito de propiciar um autoconhecer, e ao mundo ao qual pertence. Nestas relações é imperativo compreender qual é o papel do cidadão no que tange seus direitos e deveres.

Desta forma, pretende-se neste artigo analisar as relações, conceitos e conjecturas de idosos em uma ferramenta online (fórum). Este recurso foi utilizado para discutir sobre a inclusão digital de pessoas mais velhas, no intuito dos idosos se conhecerem e discutirem o seu papel nesta sociedade e na sua formação. Para tanto é necessário aprofundar, inicialmente, o conceito de auto-organização utilizado como base neste artigo.

\section{O CONCEITO DE AUTO-ORGANIZAÇÃO}

O conceito de auto-organização vem sendo discutido no decorrer dos anos e muitos especialistas possuem diferentes interpretações. Maturana e Varela (1995) discutiram sobre a autopoiese, que seria o processo de se produzir continuamente a si mesmo, uma organização na organização. Apesar dos autores utilizarem uma perspectiva biológica, é possível se pensar no desenvolvimento do conhecimento. Esta perspectiva é singular e propicia uma reflexão acerca dos aspectos voltados para a educação e será o foco neste artigo.

Morin(1999) na sua teoria considera que o conhecimento é a construção, desconstrução e reconstrução constante, em um processo recursivo, onde o aluno e o professor se autoconstroem, pois a organização de um (e suas reconstruções) afetará o outro.

Outra autora que aprofundou os seus estudos na teoria da auto-organização foi Clara Costa Oliveira.No livro "A Educação como Processo AutoOrganizativo"(Oliveira, 1999) a autora traz múltiplas reflexões sobre o assunto e sua aplicação, incluindo discussões e referenciais de autores como Piaget, Freinet entre outros. A autora traz estas referências no intuito de questionar a auto-formação dos adultos como uma prática possível. Apesar de alguns dos autores por ela (Oliveira, 1999) citados não serem estudiosos especificamente da aprendizagem de adultos, observa-se que em cada autor se encontram traços do movimento auto-organizativo.

Por exemplo, John Dewey considera que os educadores deveriam utilizar o interesse espontâneo de cada educando como forma de ação na sua busca pela aprendizagem. Ou mesmo a inter-relação entre a escola e o mundo que cerca o educando como cita Celestine Freinet na sua teoria. Portanto o processo autoorganizativo refere-se basicamente a autoformação das pessoas (Oliveira, 1999).

Nos seres humanos, que são seres vivos auto-observacionais, ocorrem processos holísticos de aprendizagem $\leftrightarrow$ educação e alguns desses processos são processos de aprendizagem $\leftrightarrow$ (auto)educação. O que ocorre é que enquanto vivemos, estamos continuamente a aprender a partir de perturbações que nos surgem nos nossos nichos, ou a partir de perturbações internas. Estas aprendizagens resultam em condutas 
diferenciadas, modos de significar o mundo pela ação que estão, por seu lado, a construir o mundo comunitário humano no qual todos fazem parte e, onde se é educado (Oliveira, 2007).Ou seja,

A aprendizagem de um adulto não pode ser apenas focalizada no contexto específico de uma sala de aulas, ou de convívio, ou de terapia. É uma atitude que tem que ser trazida para todas as atividades quotidianas em que participa (Oliveira, 1999, p.323).

Para a autora, em Oliveira (1999) a conscientização de cada um dos membros de uma comunidade de que se educa na e com a linguagem que partilham é de suma importância, pois a linguagem humana surge como um instrumento construído para a comunicação com o outro e consigo próprio. É na narrativa e na auto-observação do que se narra que há mudança na significação linguística e, ao mesmo tempo, no comportamento individual e comunitário.

Portanto, não há uma única forma de ensinar e métodos eficazes, uma vez que as relações que se criam durante a vida com o outro irão sempre confrontar e reorganizar as estruturas e significações.

\section{O MOVIMENTO AUTO-ORGANIZACIONAL NA GERONTOLOGIA}

Na gerontologia a cada ano cresce a preocupação e discussão de como proporcionar uma qualidade de vida para as pessoas que estão envelhecendo. Esta preocupação fica evidente nos congressos internacionais e nacionais que são desenvolvidos na área, tanto pela Sociedade Brasileira de Geriatria e Gerontologia (SBGG) como pela Associação Brasileira de Gerontologia (ABG).

Algumas pesquisas na área da gerontologia mostram que para se ter um envelhecimento bem sucedido depende dos papéis sociais significativos. Ou seja, aspectos como situação econômica, educação continuada e plasticidade individual podem propiciar um envelhecer com qualidade de vida (Pasqualotti, 2003).

A qualidade de vida envolve o conceito de envelhecimento ativo. Este termo refere-se à otimização do potencial social, físico e mental dos idosos ao longo da vida, a partir, por exemplo, de intervenções educativas (Tamer, Petriz, 2007; Both, Portella, 2003).

Ao longo dos anos, culturalmente, foram construídos teorias e paradigmas sobre a educação de adultos. Infelizmente o processo de educação de pessoas mais velhas foi abandonado durante muitas discussões que ocorreram, sendo retomado há pouco tempo.

Apesar de haver um consenso em algumas populações em considerar que o processo de aprendizagem limita-se apenas as faixas etárias escolares, alguns estudos possuem outra abordagem. O novo paradigma emergente sobre o envelhecer prioriza a oportunidade de uma aprendizagem em todas as idades para atualização e crescimento pessoal. O que irá permitir a possibilidade de assumir responsabilidades nas diversas épocas da vida (Osório, 2007; Oliveira, 1999; Oliveira, 2007; Delors, 1996).

A aprendizagem pode acontecer ao longo da vida, e não apenas em um período da vida pessoal; e na "largura" da vida, não apenas no sistema escolar (Martín, 2007; Vallespir, Morey, 2007; Scortegagna, 2006, Delors, 1996).

Partindo da premissa que é necessário investir na educação, muitos estudos estão sendo desenvolvidos para melhorar a qualidade do processo de ensino e aprendizagem. A teoria da auto-organização poderia auxiliar neste processo, uma vez 
que os idosos estão preocupados em resolver problemas de atividades de vida diária, podendo esta auto-organização estimular os seres humanos a pensarem e encontrarem soluções para problemas cada vez mais complexos da vida.

As intervenções socioeducativas contribuem muito na autoconfiança para o enfrentamento de situações, principalmente novas, por motivar e possibilitar uma auto avaliação e autovalorização (Martín, 2007; Azevedo E Souza, 2003). Ou seja, as intervenções educativas possibilitam ao idoso o autoconhecimento por ser uma forma de propiciar a sua confiança em seu potencial. E a tecnologia poderá ser um grande aliado para esta autovalorização.

É fundamental propiciar a produtividade e revelar os potenciais dos idosos, incluindo a utilização das tecnologias como forma de desenvolver perspectivas técnicolaborais (adaptação às mudanças) e humanas (comunicação), a fim de reduzir a segregação e isolamento (Osório, 2007).

Para uma melhor compreensão sobre a relação da auto-organização e as tecnologias, a seguir será abordada a metodologia adotada neste artigo.

\section{METODOLOGIA}

O presente artigo tem como base um projeto desenvolvido na Universidade Federal do Rio Grande do Sul denominado "Introdução da Educação a Distância para idosos". O referido projeto pretende propiciar a capacitação de pessoas com mais de 60 anos no uso de tecnologias de informação e comunicação utilizadas na Educação a Distância (EAD). A partir de cursos de extensão, objetiva-se que os idosos desenvolvam competências necessárias para participação de cursos na modalidade online.

No decorrer do projeto, foram utilizados recursos dos ambientes virtuais de aprendizagem ${ }^{1}$ ROODA (Rede cOOperativa De Aprendizagem) como: webfólio, batepapo, diário de bordo e fórum. Sendo este último utilizado para análise de dados sobre a auto-organização.

Desta forma, a metodologia foi desenvolvida em uma abordagem quantitativa e qualitativa. Este formato foi eleito pela viabilidade na complementação dos dados, a fim de auxiliar na compreensão do objeto de estudo. Em relação a aspectos qualitativos foi utilizada uma abordagem interpretativa para auxiliar o processo de compreensão dos dados que foram coletados no decorrer da pesquisa.

A coleta de dados foi realizada a partir de observações participantes e produção intelectual dos idosos de um fórum realizado no ano de 2011durante o desenvolvimento do curso.

Em relação aos dados qualitativos do fórum foram utilizados os passos sugeridos por Bardin (2004) para a análise das narrativas realizadas. Já em relação aos aspectos quantitativos a análise foi a partir da distribuição de frequência representada em percentuais, média e desvio padrão. Este tipo de análise foi utilizado principalmente para delinear o perfil do público participante.

No ano de 2010, inicialmente, foram selecionados aleatoriamente20 idosos a partir de uma lista de inscritos de 200 pessoas mais velhas. Esta lista foi formada a partir de contato telefônico realizado pelos idosos interessados no anúncio vinculado em um jornal gaúcho no mesmo ano. Os idosos selecionados deveriam ser compatíveis com os seguintes pré-requisitos: ter 60 anos ou mais, ser alfabetizado, ter conhecimento

\footnotetext{
${ }^{1}$ Um ambiente virtual de aprendizagem é definido como uma plataforma composta de infraestrutura tecnológica que possibilita a comunicação, interação e interatividade dos atores (alunos, professores, tutores, designers entre outros), objetivando o ensino e aprendizagem (Behar, 2009).
} 
básico de informática. Em relação ao número total de alunos selecionados, apenas 16 finalizaram o semestre e participaram do fórum de discussão, sendo estes os analisados neste artigo.

As aulas do curso foram desenvolvidas uma vez por semana, com duração de duas horas cada em um laboratório de informática. Durante o desenvolvimento do curso foram utilizados recursos da web e ferramentas do ambiente virtual de aprendizagem (AVA) ROODA como apoio para o desenvolvimento do curso, principalmente para a realização das atividades fora do horário de aula presencial, como foi o caso do fórum, recurso escolhido para análise. Diferentes formas de comunicação virtuais foram realizadas durante o curso, inclusive outros fóruns foram realizados além do selecionado neste artigo para a análise dos dados.

Entre os fóruns realizados durante o curso, se destacou o fórum sobre "Benefícios da Inclusão Digital". Este fórum pretendia discutir qual é a visão que os idosos participantes possuíam sobre cursos de inclusão digital para idoso e qual a sua relação com o cotidiano dos mesmos. Desta forma, a seguir serão apresentados e discutidos os dados coletados durante o processo.

\section{RESULTADOS E DISCUSSÃO DOS DADOS}

Como já foi dito em seções anteriores, a ferramenta utilizada para coleta das narrativas realizadas pelos idosos foi um fórum online disponível no ambiente virtual de aprendizagem (AVA) ROODA. Considera-se um fórum como sendo uma forma de comunicação assíncrona, ou seja, não é necessário estar ao mesmo tempo e no mesmo local para realizar tal forma de interação. A característica fundamental do fórum é a possibilidade de realizar interações e discussões entre os participantes de um AVA, havendo sempre um tema gerador. $O$ fórum possibilita uma narrativa entre os participantes, o que para Oliveira (1999) é considerado um dispositivo para a autoorganização.

\footnotetext{
Diferentes estados de ânimo perpassam nas narrativas, sendo possível identificar sentimentos de desconfiança, de revolta, de não resistência à frustração, de baixa autoestima, de incapacidade em confiar nos outros entre outros (Oliveira, 2007, p. 178).
}

Desta forma, em relação à participação dos idosos no fórum, dos 16 alunos apenas 10 deles participaram do mesmo. $\mathrm{O}$ fórum foi aberto pelo professor responsável no início do semestre e ficou a disposição dos alunos idosos até o fechamento do semestre, totalizando 21 postagens realizadas.

O grupo de 16 idosos possui uma média de idade de 67 anos, sendo 4 do sexo masculino. Já em relação à escolaridade, predomina o ensino superior completo (44\%), seguido do ensino médio (38\%). Estes dados mostram um nível de escolaridade alta no grupo de alunos.

Após a leitura das 21 postagens realizadas no fórum, foi possível verificar padrões que se repetiam com mais intensidade nas respostas dos alunos idosos, destacando uma preocupação com questões de saúde e cidadania. Apesar de não haver a possibilidade de separação de tais padrões localizados, optou-se por separá-los para que haja uma melhor discussão dos dados coletados.

\subsection{Cidadania}


Quando foi verificado um padrão nas narrativas dos idosos, observou-se que os mesmos relacionaram muitas de suas escritas com questões sobre estar atualizado, e em constante comunicação com as pessoas. Desta forma, optou-se por denominar de "cidadania", uma vez que os mesmos procuraram estes cursos para se tornarem mais presentes na sociedade, ou seja, tornarem-se cidadãos ativos. Ao longo das discussões do fórum, os idosos relataram que a inclusão digital é importante para estarem constantemente atualizados com os novos padrões que a sociedade atual impõe ("Se $o$ idoso não procurar atualizar-se com as ferramentas do mundo moderno, estará fadado ao isolamento", "A inclusão digital nos beneficia com informação rápida e ampliação das redes de relacionamento entre muitas outras", "Toda esta tecnologia nos possibilita manutenção social, do exercício da cidadania, autonomia, acesso a uma sociedade dinâmica e ao mesmo tempo complexa", "Após ler, as abordagens que os colegas fizeram sobre a inclusão digital na terceira idade, concluo que, "não podemos viver sem estarmos conectados"”).

É possível observar nos diálogos que os idosos sabem que as tecnologias podem propiciar uma atualização a qual não estavam acostumados, e cada vez mais é uma necessidade emergente a alfabetização com as tecnologias. Claro que cabe ao educador estar consciente das relações realizadas pelos idosos do "passado" com o "presente". É relevante ao se trabalhar com este assunto que não apenas o presente é o mais importante, mas também todo o aprendizado anterior poderá auxiliar na compreensão do "novo" (tecnologias).

Remetendo a teoria da auto-organização, é necessário considerar os "acoplamentos" dos organismos, ou seja, o processo de educação a que um organismo (humano) se encontra sujeito deverá ser analisado e compreendido tendo em conta os acoplamentos estruturais que ele vai realizando com outros organismos, e, portanto tendo também em conta o processo de educação a que ele vai sujeitando os outros organismos com que ele está em processo de acoplamento (Oliveira, 1999). Cabe salientar que quando remetemos a palavra organismo se refere não apenas a questões moleculares, mas também sociais.

Outro ponto lembrado pelos idosos no fórum foi relacionado com a comunicação, onde segundo os mesmos as tecnologias possibilitaram uma maior interação com o "mundo" ("Para nós os idosos, eu considero um excelente meio de comunicação, com pouco custo e me mantem bastante ativa", "Os benefícios da inclusão digital são inúmeros. Tanto no aspecto psicológico de aproximação virtual de pessoas queridas, como na possibilidade de iniciar novos relacionamentos", "É através do computador que eu interajo com um maravilhoso grupo de pessoas da Alemanha").

Dois idosos comentaram que uma das grandes barreiras na aprendizagem está relacionada com a falta de motivação que a própria família incute no idoso quando estes desejam aprender a utilizar as tecnologias ("Faltando muitas vezes o incentivo da família", "Eu concordo parcialmente com os colegas quanto aos impedimentos de idosos utilizarem a informática, além do medo do novo, e de serem ridículas pq não entendem e serem obrigadas a perguntar, e muitas vezes os familiares não terem paciência p/explicar”).

O processo de aprendizagem com pessoas mais velhas fica prejudicado quando educador não incorpora os padrões de compreensão do mundo do idoso. Se isto não ocorrer os educandos acabam eliminando a aprendizagem pontual que ocorreu (Oliveira 2007).Ou seja, a contemporaneidade está exigindo a não omissão do papel observacional das descrições de quaisquer fenômenos de aprendizagem e de educação. Portanto, ir além dos simples conteúdos programáticos que as escolas impõem, mas incentivar a educação permanente em todas as idades, isto é, ocorrendo ao longo de toda 
a existência e em qualquer contexto no qual se viva (Oliveira, 2009). Como cita uma idosa: "Para mim é impossível viver sem tecnologia, quero estar cognitivamente ativa, a vida é movimento não podemos parar".

\subsection{Saúde}

Além da preocupação dos idosos acerca de serem cidadãos ativos, outro fator muito citado durante a discussão do fórum foram assuntos relacionados à saúde. Muitos idosos, como serão contemplados a seguir, relacionaram a inclusão digital com preocupações sobre atividades saudáveis, psicológicas ou mesmo corporais.

É interessante analisar que os idosos estão preocupados com aspectos de saúde quando se fala em inclusão digital. De acordo com os eles, é necessário não desconsiderar a realização de exercícios físicos, conforme as falas ("Mas acho que além da comunicação não devemos deixar de exercitar o corpo, porque não se vive só com a mente", "Tem um inconveniente estamos ficando muito tempo sentados na frente do computador", "Muitas vezes a boa leitura e uma boa caminhada ao ar livre e um bom papo com amigos também é necessário", "Estarmos atentos a nossa postura frente ao computador. Fazer exercícios físicos", "Mas também devemos cuidar com os prejuízos como: isolamento, postura e falta de exercícios físicos", "Vamos apenas ter cuidado em usá-las sem prejudicar nossa saúde”, "Também não devemos de esquecer de trabalhar nosso corpo com exercícios de coordenação motora, alongamento $e$ ginástica, que assim estaremos completos para uma vida melhor”).

Outro ponto abordado pelos idosos é o benefício da inclusão digital para as pessoas com necessidades físicas, como ("Sem falar nos benefícios psicológicos aproximando pessoas distantes fisicamente, aumentando a autoestima de todos", " $E$ também as pessoas doentes ou com deficiências físicas de qualquer idade facilitam a sua vida através da internet", "É tudo maravilhoso mantendo a nossa mente ativa" em relação às tecnologias).

A preocupação de estarem mentalmente ativos é outro fator considerado pelos idosos ("O nosso cérebro não envelhece nunca a não ser que nós permitamos que ele atrofie por falta de uso", "E para nós é muito importante, de essa maneira seguimos mantendo a mente ativa", "Esta é uma atividade muito gratificante, além de me deixar muito ativa”).

Os idosos sabem das suas limitações orgânicas e as consideram quando se trata da inclusão digital ("Dentre os motivos que impedem os idosos de procurar buscar novos conhecimentos, está a aversão a informática, que para muitos é considerada 'um bicho papão', algo que principalmente, se danifica e estraga, decorrente das suas limitações físicas decorrentes da idade", "Apesar de termos um pouco mais dificuldades que os jovens, nós tb chegamos lá. A única diferença é que o nosso ritmo é mais lento").

Podemos observar pelos relatos apresentados que os idosos estão no processo de envelhecimento ativo (WHO, 2002), pois, os conceitos abrangentes do tipo "guardachuva" como o de qualidade de vida, apontam para a necessidade de considerar os aspectos valorizados pelos mesmos para o bem-estar global como a saúde, mas também a satisfação com a vida e o bem-estar psicológico, assim como o ambiente social e físico em que estão inseridos. E todas estas reflexões só puderam ser realizadas por meio de um processo de auto-organização que se deu na interação com o fórum proposto no curso. 


\section{CONCLUSÃO}

Partindo dos dados discutidos é possível verificar que a inclusão digital para idosos remete a preocupações como saúde e cidadania. Quando o tema é debatido, outros fatores antes desconsiderados, como as interações anteriores no curso, são considerados e utilizados a favor do processo de aprendizado. Neste artigo foi possível observar que existe uma auto-organização no que tange o uso das tecnologias pelos idosos, uma vez que ferramentas de comunicação/discussão, como o fórum, possibilitaram uma reflexão acerca da aprendizagem individual. Esta análise individual presente nas narrativas e contrapontos dos idosos, poderá levar o próprio ao autoconhecimento e, uma vez entendido todo o seu processo, acarretará na compreensão das estruturas organizativas (biológicas ou não). Estas análises poderão possibilitar a compreensão mais aprofundada do processo de aprendizagem e de existência enquanto ser humano que faz parte do mundo.

Conforme escreveu um dos idosos no fórum: "Uma maquina Por mais "inteligente" que seja, nunca substituirá eficazmente a atividade mental e criativa do homem", o que mostra os processos complexos que podem possibilitar ao idoso uma autovalorização do seu potencial.

\section{REFERÊNCIAS BIBLIOGRÁFICAS}

AZEVEDO E SOUZA, Valdemarina Bidone. A motivação do idoso para reaprender a aprender: um desafio para propostas de intervenção educativa. In: TERRA, Newton Luiz; DORNELLES, Beatriz. Envelhecimento bem-sucedido. Porto Alegre: EDIPUCRS, 2003.

BARDIN, Lawrence. Análise de Conteúdo. Lisboa: Portugal. 2004.

BEHAR, P (col.). Modelos Pedagógicos em Educação a Distância. Porto Alegre: Artmed, 2009.

BOTH, Agostinho; PORTELLA, Rodrigues. Gerontogogia: uma proposta socioeducativa para idosos. In: BOTH, Agostinho; BARBOSA, Marcia Helena S.; BENINCÁ, Ciomara Ribeiro S. Envelhecimento humano: múltiplos olhares. Passo Fundo: UPF, 2003.

DELORS, Jacques (coord.). Os quatro pilares da educação. In: UNESCO. Educação: um tesouro a descobrir. São Paulo: Cortez, 1996

MARTÍN, Antonio Víctor. Gerontologia educativa: enquadramento disciplinar para o estudo e intervenção socioeducativo com idosos. In: OSÓRIO, Agustín Requejo; PINTO, Fernando Cabral. As pessoas idosas: contexto social e intervenção educativa. Lisboa: Instituto Piaget, 2007.

MATURANA, Humberto, VARELA, Francisco. A Árvore do Conhecimento.

Campinas: Editorial Psy, 1995.

MORIN, Edgar. O método 3: O conhecimento do conhecimento. Porto Alegre: Sulina, 1999.

OLIVEIRA, Clara Costa. Humanidades na Formação Médica: Realidade ou Farsa? Reflexão e Acção, v. 17, N.2. 2009. p.225-242.

OLIVEIRA, Flávia da Silva; OLIVEIRA, Rita de Cássia. As pessoas idosas no Brasil: contexto demográfico, político e social. In: OSÓRIO, Agustín Requejo; PINTO, Fernando Cabral. As pessoas idosas: contexto social e intervenção educativa. Lisboa: Instituto Piaget, 2007. 
OLIVEIRA. Clara Costa. Educação como processo auto-organizativo. Lisboa: Instituto Piaget, 1999.

OLIVEIRA. Clara Costa. Contributos Educativos e Comunitários do Movimento Epistemológico da Auto-organização - Um método Auto-organizativo na formação de educadores de adultos. Educação, ano XXX, n. 1 (61), p. 165-180, jan./abr. 2007

OSÓRIO, Agustín Requejo. Os idosos na sociedade actual. In: OSÓRIO, Agustín Requejo; PINTO, Fernando Cabral (orgs). As pessoas idosas: contexto social e intervenção educativa. Lisboa: Instituto Piaget, 2007.

PASQUALOTTI, Adriano. Desenvolvimento dos aspectos sociais na velhice: experimentação de ambientes informatizados. In: BOTH, Agostinho; BARBOSA, Marcia Helena S.; BENINCÁ, Ciomara Ribeiro S. Envelhecimento humano: múltiplos olhares. Passo Fundo: UPF, 2003.

SCORTEGAGNA, Helenice de Moura. Oficinas temáticas como estratégia pedagógica. In: CASARA, Miriam Bonho; CORTELLETTI, Ivonne A.; BOTH, Agostinho. Educação e envelhecimento humano. Caxias do Sul: EDUCS, 2006.

TAMER, Norma Liliana; PETRIZ, Graciela. A qualidade de vida dos idosos. In: OSÓRIO, Agustín Requejo; PINTO, Fernando Cabral. As pessoas idosas: contexto social e intervenção educativa. Lisboa: Instituto Piaget, 2007.

VALLESPIR, Jordin; MOREY, Mercè. A participação dos idosos na sociedade: integração vs. Segregação. In: OSÓRIO, Agustín Requejo; PINTO, Fernando Cabral. As pessoas idosas: contexto social e intervenção educativa. Lisboa: Instituto Piaget, 2007.

VITOLA, Janice; ARGIMON, Irani de Lima. Aspectos psicológicos do envelhecimento. In: TERRA, Newton Luiz; DORNELLES, Beatriz. Envelhecimento bem-sucedido. Porto Alegre: EDIPUCRS, 2002.

WHO/NMH/NPH. Active ageing: a policy framework. Geneve: World Health Organization, 2002. 